\title{
GENERACION DE SUPERFICIES CURVAS UTILIZANDO EL PROGRAMA CADET
}

\author{
(CURVED SURFACES GENERATION USING THE CADET PROGRAM)
}

\author{
Alfonso Recuero, Olga Río y José Pedro Gutiérrez, Drs. Ings. de Caminos \\ IETCC/CSIC. Madrid
}

$403-10$

\section{RESUMEN}

En el presente trabajo se muestra una extensión del programa CADET, para el dibujo de perspectivas de objetos tridimensionales, mediante la cual es posible generar contornos planos descritos en su propio plano y que posteriormente sirven de base en la definición de primitivas complejas. Estos contornos pueden representar las meridianas de superficies de revolución, la directriz de superficies cilíndricas o cónicas, los bordes de elementos planos complicados, etc. Se incluyen los listados en HP-BASIC de las subrutinas de generación y dibujo de estos contornos, asi como una descripción detallada de su utilización con un objeto completo.

\section{SUMMARY}

In this paper an extension to the CADET program, for the perspective drawing of 3-dimensional objects, is described. By means of this extension playing contours can be defined in their plane, which can subsequently be used in the generation of complex primitives. This contours may define the meridian curve of surfaces of revolution, the directive curve of conical or cilindrical surfaces, the borders of complicate plane elements, etc. The listings of the subroutines, written in HP-BASIC, are included together with a comprehensive explanation of how to use it in generating a complet object.

\section{INTRODUCCION}

El programa CADET para el dibujo de perspectivas de objetos tridimensionales con ayuda de un computador, ha sido ya exhaustivamente descrito en (1), donde pueden encontrarse los listados completos del programa, así como ejemplos de su aplicación. En dicha publicación se indica que las posibilidades de CADET pueden ser ampliamente mejoradas por sus usuarios, especialmente incorporándole nuevas formas de definir los objetos a dibujar.

En el presente trabajo se muestra la forma de generar contornos planos descritos en su propio plano, los cuales pueden emplearse para definir elementos planos de forma complicada, la meridiana de superficies de revolución, las directrices de superficies cilíndricas o cónicas, etc.

Se incluyen los listados en HP-BASIC de las subrutinas de generación y dibujo de estos contornos, así como una descripción detallada de su utilización con un objeto completo.

\section{EL PROGRAMA CADET}

El programa CADET permite el dibujo asistido por computador de perspectivas de objetos tridimensionales. Este programa, escrito en BASIC para ordenadores HP de las Series 200 y 300 , ha sido desarrollado en el Instituto Eduardo Torroja de la Construcción y del Cemento, perteneciente al Consejo Superior de Investigaciones Científicas.

El programa permite mantener en memoria un conjunto de objetos que pueden manejarse independientemente. Así, es posible trasladar, girar, distorsionar o eliminar un solo objeto, fundir dos objetos en uno sólo, duplicar objetos existentes en otra posición o traspasar elementos de un objeto a otro. Las perspectivas y la iluminación se hacen siempre con el conjunto.

Los objetos a dibujar se discretizan mediante elementos rectilíneos y elementos poligonales planos de forma cualquiera, simple o múltiplemente conexos. El esquema de almacenamiento 
utilizado es extremadamente compacto y reduce al mínimo la capacidad de memoria central necesaria.

La definición de los objetos se hace de forma interactiva, pudiendo definir y modificar las coordenadas de los vértices y la descripción de los elementos.

Incluye una variada gama de primitivas complejas que permiten definir elementos planos cualesquiera, superficies de revolución, superficies cilíndricas o cónicas, paraboloides hiperbólicos, pórticos regulares de edificación, etc. También es posible asignar espesor a los elementos planos, construyendo prismas, tomando como base dichos elementos. Pueden, también, eliminarse los datos redundantes o no utilizados, consecuencia de generaciones automáticas, cortes, etcétera.

Se determinan automáticamente las intersecciones de partes llenas de elementos planos, lo cual simplifica notablemente la definición de los objetos. Es posible almacenar en archivos de disco objetos completos y recuperarlos en cualquier posición del espacio, pudiendo, de este modo, crear y manejar catálogos de elementos de uso frecuente o repetitivo.

Un objeto puede ser seccionado por un plano, eliminando la parte del mismo que quede a un lado del plano de sección. Asimismo, el conjunto de objetos puede ser iluminado por un haz de luz paralela a una dirección cualquiera, determinando los contornos de la sombra arrojada por los objetos sobre sí mismos y sobre el plano de tierra.

Permite dibujar cualquier tipo de perspectiva del conjunto de objetos, tanto de tipo axonométrico como cónicas, y situar al espectador en cualquier posición del espacio, incluso, en las perspectivas cónicas, dentro del objeto. En el caso de perspectivas cónicas, permite definir, además, el campo de visión, obteniendo efectos de teleobjetivo, gran angular, etc. Las líneas ocultas a la visión del espectador pueden omitirse o dibujarse de puntos. Además, pueden definirse como transparentes a la visión del espectador los elementos planos que se desee, con el fin de poder observar partes interiores del objeto.

Es posible seleccionar el ángulo mínimo que deben formar dos elementos planos con una arista común para que la misma sea considerada redondeada, esto es, que sólo sea dibujada cuando forme parte del contorno aparente del objeto, de acuerdo con la posición del espectador. Esto permite, en el caso de superficies curvas, dibujar exclusivamente los contornos aparentes de las mismas o el esqueleto de elementos utilizados en su discretización.
Permite almacenar, en archivos de disco, dibujos completos y recuperarlos posteriormente. Asimismo, permite ampliar las partes del dibujo que se deseen.

Los dibujos pueden presentarse en la pantalla gráfica o en un dispositivo trazador gráfico o Plotter. Los algoritmos utilizados para determinación de intersecciones de elementos, dar cortes al objeto, determinar sombras arrojadas, limitar el campo de visión, almacenar dibujos realizados, o determinar las partes vistas u ocultas del objeto, son originales de los autores.

Se han utilizado, asimismo, procedimientos del tipo minimax con el fin de acelerar los procesos de cálculo, eliminando operaciones innecesarias.

\section{DEFINICION DE VERTICES SOBRE UN PLANO}

Cuando se trata de situar en el espacio un conjunto de puntos coplanarios es más sencillo definir estos puntos en un sistema de coordenadas locales en el plano que los contiene y situar en el espacio este sistema local. Esto resulta particularmente útil en la definición de elementos planos de forma complicada, la meridiana de superficie de revolución, la directriz de superficies cilíndricas o cónicas, etc.

El programa permite generar una secuencia de puntos sobre un plano, pudiendo añadir puntos, modificar las coordenadas de puntos existentes, definir puntos sobre un arco de circunferencia, insertar o eliminar cualquier número de puntos; trasladar, girar alrededor de un punto o deformar las coordenadas de cualquier parte de la secuencia (ver subrutina CPDEF). Para visualizar mejor la posición de los puntos (ver subrutina CPDIB) se presentará en pantalla una línea poligonal uniendo los puntos de la secuencia. Una vez dada por válida la secuencia de puntos, la posición del plano en el espacio se fija mediante las coordenadas globales del origen local, las coordenadas de un punto del eje X local positivo y las componentes de un vector normal al plano.

El programa conserva la definición de la última secuencia de puntos sobre un plano que puede ser nuevamente llamada para su modificación o cambio de la situación del plano. En la utilización de estos puntos, para definir elementos planos, meridianas de superficies de revolución, etc., podrá utilizarse cualquier fragmento de la sucesión de puntos definida.

Cabe señalar que el procedimiento utilizado para definir las coordenadas de los puntos es analitico, no obstante es muy simple modificarlo de modo que, el programa pueda leer las coordenadas de un dispositivo captador externo como 
puede ser una tableta gráfica. Esta modificación potenciaría notablemente las posibilidades del programa al poder introducir los datos a partir de planos del objeto.

\section{EJEMPLO DE UTILIZACION}

Para demostrar la forma de utilización del programa se va a describir a continuación una secuencia completa de operaciones con el programa que permite dibujar el objeto de la figura 1.

El objeto en cuestión podría ser un codo a $90^{\circ}$ de una conducción de alcantarillado. En la figura 1 se muestran sendas perspectivas cónicas con tres puntos de fuga del objeto, una en la que se han dibujado las líneas ocultas y otra en la que éstas se han suprimido.

En primer lugar, se define el contorno de la sección de la alcantarilla en su plano. Para ello se ha
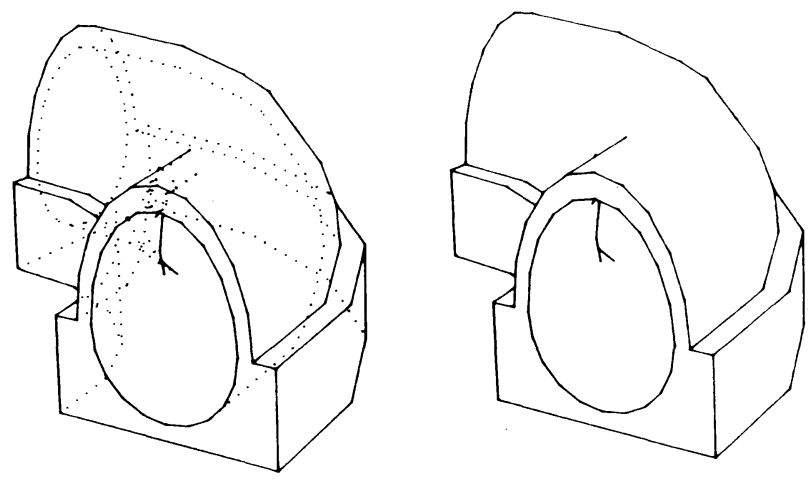

Fig. 1

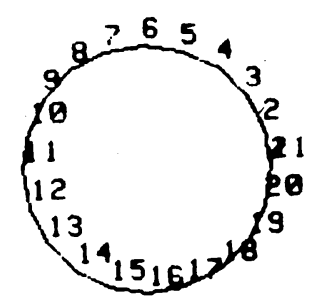

Fig. 2

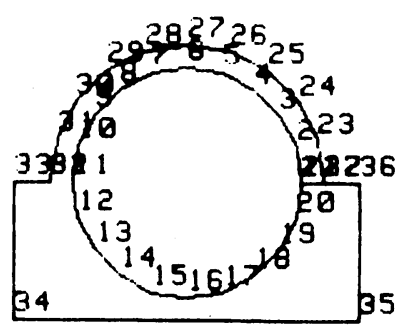

Fig. 5

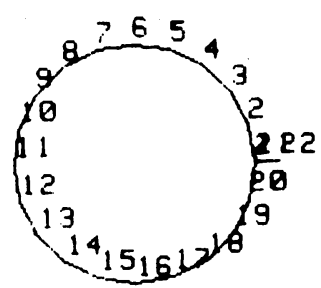

Fig. 3

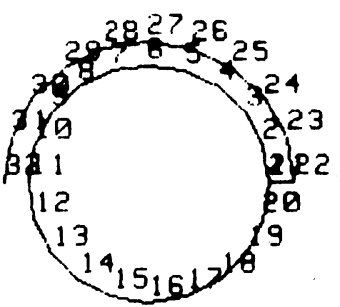

Fig. 4

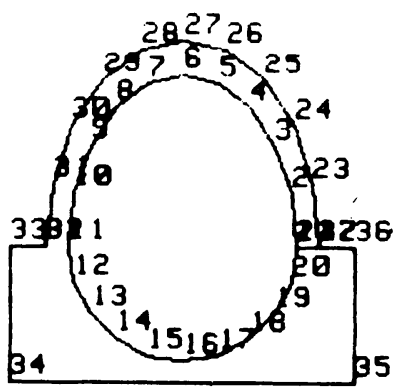

Fig. 7

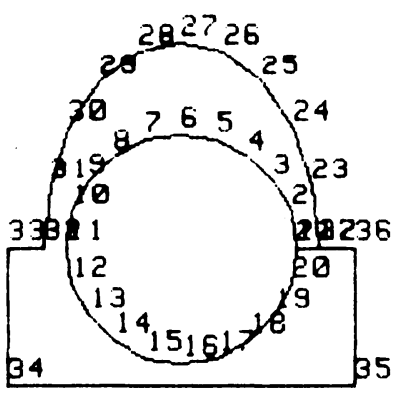

Fig. 6 empezado por situar el eje $\mathrm{Y}$ del sistema de coordenadas local en el eje de revolución de las superficies. Se define entonces una circunferencia cuya parte inferior coincide con el semicírculo inferior del tubo interior, utilizando para ello 20 tro-

A continuación se define un nuevo punto (22) que corresponde al arranque de la superficie curva exterior (ver Fig. 3).

A continuación, se genera una semicircunferencia concéntrica con la primera y definida por 10 trozos - puntos 22 a 32- (ver Fig. 4).

Seguidamente se completa la definición de la base rectangular inferior de la sección - puntos 32 a $37-$ (ver Fig. 5).

Una vez definda esta sección auxiliar deben deformarse las semicircunferencias superiores corespondientes a las partes altas exterior e interior del tubo. En primer lugar se deforma la parte de la secuencia, entre los vértices 22 y 32 tomando como punto fijo el centro de la circunferencia y dando como factores de dilatación según las direcciones $\mathrm{X}$ e $\mathrm{Y}, 1$ y 1,5 respectivamente (ver

Por último, se deforma de modo análogo al caso anterior, la secuencia de puntos entre el 1 y el 11 (ver Fig. 7). Con lo cual, queda definida completamente la sección transversal del tubo por una secuencia de 37 puntos cuyas coordenadas pueden verse en la tabla 1. 


\begin{tabular}{|c|c|c|}
\hline \multicolumn{3}{|c|}{$\begin{array}{l}\text { Tabla I } \\
\text { Coordenadas de los puntos } \\
\text { del contorno plano }\end{array}$} \\
\hline Punto & Coordenada X & Coordenada Y \\
\hline $\begin{array}{r}1 \\
2 \\
3 \\
4 \\
5 \\
6 \\
7 \\
8 \\
9 \\
10 \\
11 \\
12 \\
13 \\
14 \\
15 \\
16 \\
17 \\
18 \\
19 \\
20 \\
21 \\
22 \\
23 \\
24 \\
25 \\
26 \\
27 \\
28 \\
29 \\
30 \\
31 \\
32 \\
33 \\
34 \\
35 \\
36 \\
37\end{array}$ & $\begin{array}{l}6.00 \\
5.95 \\
5.81 \\
5.59 \\
5.31 \\
5.00 \\
4.69 \\
4.41 \\
4.19 \\
4.05 \\
4.00 \\
4.05 \\
4.19 \\
4.41 \\
4.69 \\
5.00 \\
5.31 \\
5.59 \\
5.81 \\
5.95 \\
6.00 \\
6.20 \\
6.14 \\
5.97 \\
5.71 \\
5.37 \\
5.00 \\
4.63 \\
4.29 \\
4.03 \\
3.86 \\
3.80 \\
3.50 \\
3.50 \\
6.50 \\
6.50 \\
6.20\end{array}$ & $\begin{array}{r}0.00 \\
0.46 \\
0.88 \\
1.21 \\
1.43 \\
1.50 \\
1.43 \\
1.21 \\
0.88 \\
0.46 \\
0.00 \\
-0.31 \\
-0.59 \\
-0.81 \\
-0.95 \\
-1.00 \\
-0.95 \\
-0.81 \\
-0.59 \\
-0.31 \\
-0.00 \\
0.00 \\
0.56 \\
1.06 \\
1.46 \\
1.71 \\
1.80 \\
1.71 \\
1.46 \\
1.06 \\
0.56 \\
0.00 \\
0.00 \\
-1.20 \\
-1.20 \\
0.00 \\
0.00\end{array}$ \\
\hline
\end{tabular}

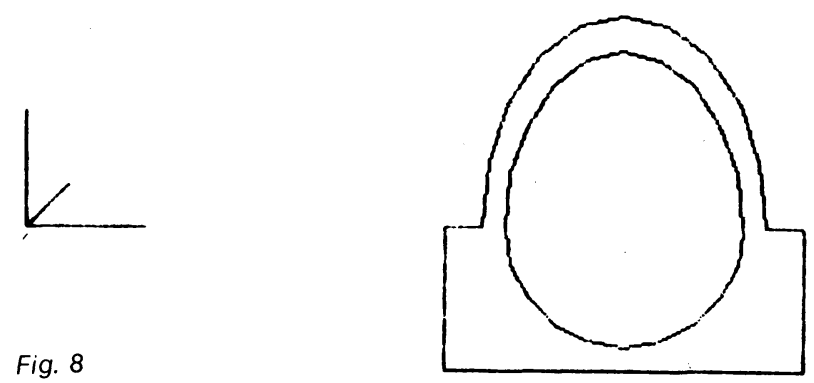

Fig. 8

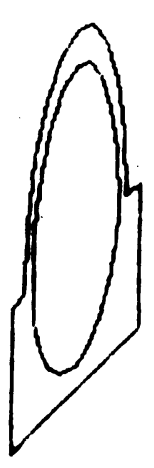

Fig. 9
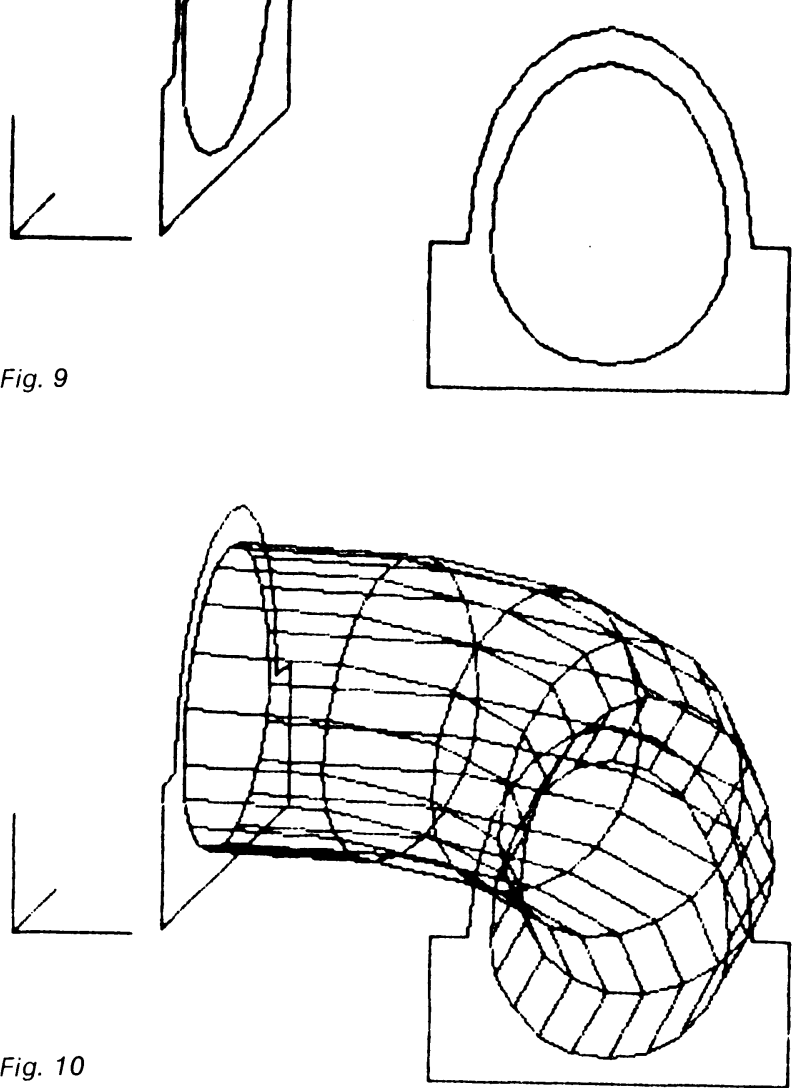

Se procede entonces a definir los elementos planos en que se va a discretizar el objeto para su dibujo. En primer lugar, se definió la sección frontal, con dos bordes, el primero de ellos definido por los puntos 22 a 36 y el segundo por los vértices 1 a 20 y situando el sistema local en el origen global coincidiendo el eje $X$ local con el eje $X$ global $y$ el eje $Y$ local con el eje $Z$ global (ver Fig. 8).

En segundo lugar, se definió la sección dorsal del tubo, de modo análogo a la sección frontal, pero haciendo coincidir en este caso el eje $X$ local con el eje $Y$ global (ver Fig. 9).

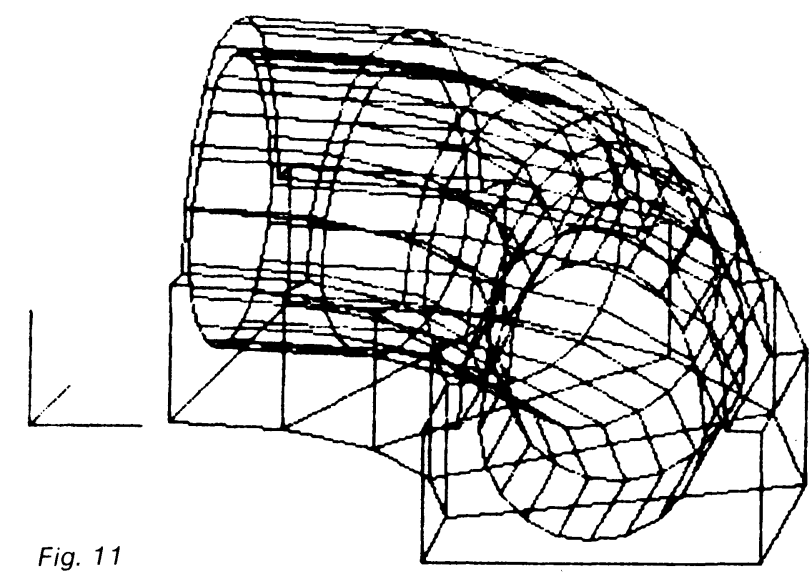

(c) Consejo Superior de Investigaciones Científicas 


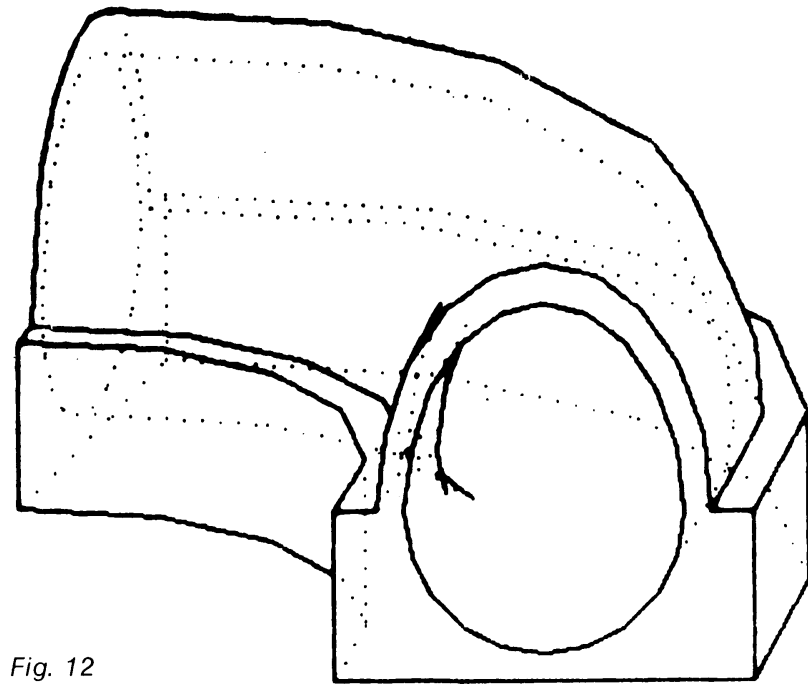

A continuación se definió la superficie interior del tubo, con una superficie de revolución que tiene como meridiana la secuencia de puntos 1 a 21 , como eje de giro el eje $\mathrm{Z}$ global y un ángulo de giro de $90^{\circ}$ dividido en 5 trozos (ver Fig. 10).

Por último se generó la superficie exterior del tubo de manera análoga al caso anterior pero utilizando entonces los puntos del 22 al 37 de la secuencia (ver Fig. 11).

Esta última figura muestra una perspectiva cabaIlera del esqueleto completo de los elementos utilizados en la discretización del objeto, considerados todos ellos como transparentes. Antes de proceder al dibujo de perspectiva del objeto es conveniente hacer una compactación de los datos, lo cual elimina los posibles vértices generados pero no utilizados y asigna una numeración única a aquellos vértices que hubieran sido numerados varias veces. Esto hace que el programa pueda reconocer todas las aristas que

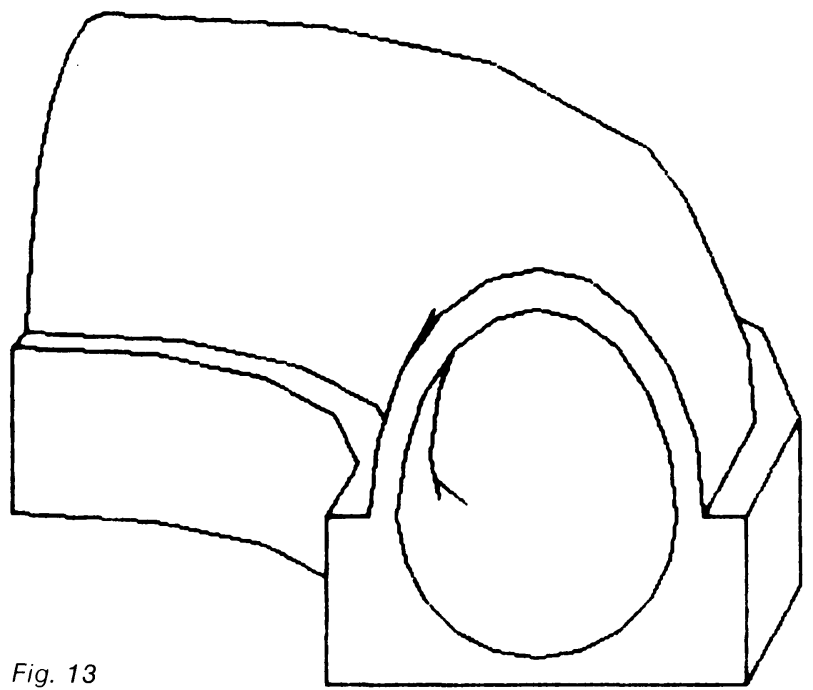

pertenezcan a más de un elemento y evitar su dibujo si fuesen redondeadas y no perteneciesen al contorno aparente.

Finalmente, en las figuras 12 y 13 , pueden verse sendas perspectivas caballeras del objeto que coinciden con la mostrada en la figura 11, pero en las que no aparecen las aristas redondeadas. En la figura 12 se manifiestan las líneas ocultas en tanto que en la 13 éstas han sido suprimidas.

\section{BIBLIOGRAFIA}

1. RECUERO,A.; GUTIERREZ, J.P.; RIO, O.: «Dibujo de perspectivas, asistido por computador, utilizando dispositivos trazadores gráficos". Monografía n. ${ }^{\circ} 380$ del Instituto Eduardo Torroja de la Construcción y del Cemento. Madrid, 1987 (en prensa).

2. RECUERO, A.; GUTIERREZ, J.P.: «Perspective drawing of three-dimensional objects". Microsoftware for Engineers. Abril, 1987.

\section{APENDICE LISTADOS DE LAS SUBRUTINAS}

Las subrutinas ANULAKEYS y EJ y la línea ESPERA a las que se hace referencia en estos listados, y que no figuran en ellos, están contenidos en el listado completo del programa CADET que puede verse en (1).

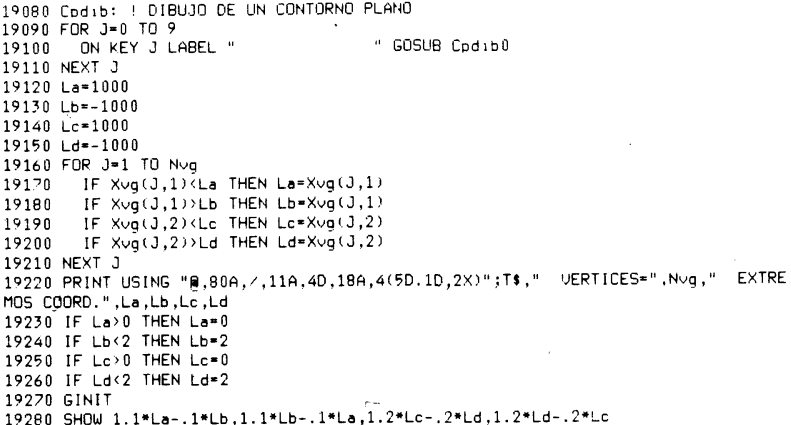

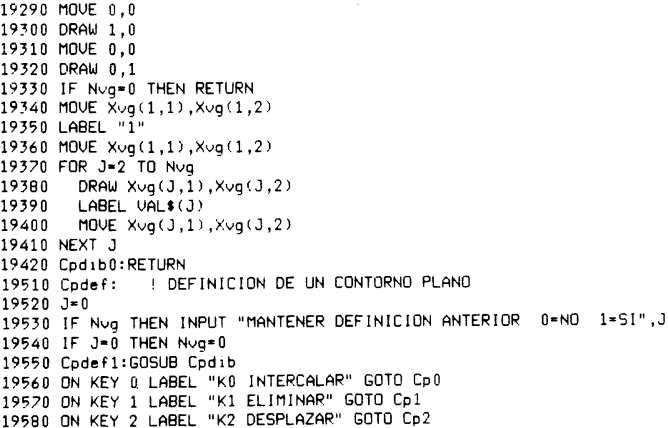


19590 ON KEY 3 LAEEL "K3 GIRAR" GOTO CP 3

19600 ON KEY 4 LABEL "K4 DEFORMAR" GOTO CP4

19620 ON KEY O LABEL "KG ARCD PUR" GOTO CP6

19630 ON KEY 7 LABEL "K? LISTADO" GOTO CP

19640 ON KEY B LABEL "KB UALE" GOTO CPB

19650 GOTO Espera

19660 CPO: INPUT "NUMERO DE PUNTOS A INTERCALAR",NI

19670 IF N1 1 THEN CPdef1, 1 PUTO POR DELANTE DEL CUăL SE DESEA INTERCALAR" J1
19680 INPUT "NUMERO DEL PUNTTO POR

19680 INPUT "NUMERO DEL PUNTO POR OE

19700 FOR J $=$ NUG TO JI STEP -1

$19710 \quad X \cup g(J+N 1,1)=X \cup g(J, 1)$
$19720 \quad X \cup g(J+N 1,2)=X \cup g(J, 2)$

19730 NEXT J

19740 FOR J-J1 TO $J 1+N 1-1$

19750 DISP "COORDENADAS $X, Y$ DEL NUEUO PUNTO";

19760 INPUT $X u g(J, 1), X v g(J, 2)$

19770 NEXT J

19780 Nug=Nug $+\mathrm{N} 1$
19790 GOTO Codef

19800 CD 1 I INPUT "NUMERO DEL PRIMER Y ULTIMO PUNTOS A ELIMINAR", J1,J2

19810 IF J1<1 OR J2) Nug OR J1>J2 THEN CPdef1

$19820 \mathrm{N1}=\mathrm{J} 2-31+1$

19830 FOR $\mathrm{J}=\mathrm{J} 2+1$ To N N

$19840 \quad X \cup g(J-N 1,1)=x \cup g(J, 1)$

19850 X X 19860 N

19870 Nug=Nug-N1

19870 Nug=Nug-N1

19890 CD2: INPUT "NUMERO DEL PRIMER Y ULTIMO PUNTOS A DESPLAZAR", J1, J2

$19900 \mathrm{IF} J 1<1$ OR J2)MV OR J1)J2 THEN CPdef

19910 INPUT "COMPONENTES DEL UECTOR DE TRASLACION", La, Lb

19920 FOR $J=J 1$ TO $J 2$

$19930 \quad X \cup g(J, 1)=X \cup g(J, 1)+L$ LO

19940 Xug( 1,2

19960 GOTO CPdef 1 "NUMERO DEL PRIMER Y ULTIMO PUNTOS A GIRAR" ,J1, J2

19980 IF J1<1 OR J2) Nug OR J1)J2 THEN CPdef 1

INPST "COORDENADAS DEL CENTRO DE GIRO",La, Lb

20000 INPUT "ANGULO DE GIRQ", LC

20010 LCELC*P $1 / 180$

$20030 \quad$ O $=X \cup(J), 1)$

$20040 \quad L 2=X \cup g(J, 2)-L b$

$20050 R=S 0 R(L 1 * L 1+L 2 * L 2)$

20060 IF $R=0$ THEN CP3

20070 IF L1 THEN Ld=ATN(L2/L1)

20080 IF $L 1=0$ AND $L 2>0$ THEN $L d=P 1$

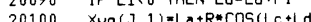

$20110 \quad x \in g(J, 2)-2$

20130 GOTO CPdef1

20140 CP4: INPUT "NUMERO DEL PRIMER Y ULTIMO UERTICE A DEFORMAR SUS COORDENADAS",

ग1, 12

20150 IF J1<1 OR J2)NUG OR J1)J2 THEN CPdef1

20160 INPUT "COORDENAOAS DEL PUNTO FIJO EN LA DEFORMACION", La , Lb

20170 INPUT "FACTORES
20180 FOR J $=\mathrm{J} 1$ TO J2

$20180 \quad X v g(J, 1)=(X \cup g(J, 1)-L a) * L C+L a$
$20200 \quad X v g(J, 2)=(X \cup g(J, 2)-L b) * L d+L b$
20210 NEXT J

20220 GOTO Cpdef 1

20240 DISP "NUMERO DEL PUNTO QUE SE DESEA DEFINIR" ;

20250 OUTPUT $2 ; 3$;

20260 INPUT $J$

20270 IF J J 1 OR J) Nug 1 THEN CPdef 1

20280 DISP "COORDENADAS DEL PUNTO"; $J ;$

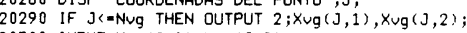

20300 INPUT Xug $(J, 1), X \cup g(J, 2)$

20310 IF J>Nug 20320 GOTO

20330 CP6: INPUT "COORDENADAS DEL CENTRO DE LA CIRCUNFERENCIA", La, LD

20340 IF Nug=0 THEN

20350 Nug=Nug.1

20360 INPUT "COORDENADAS DEL PUNTO ORIGEN DEL ARCO", Xug (Nug, 1), Xug (Nug, 2

20370 END I

20380 L L $1=x \cup g($ Nug, 1$)$-Lo

20400 IF L1 THEN Ld $=A T$ AT (L2/L1)

20410 IF L1=0 THEN Ld=PI/2

20420 IF LI<0 THEN Ld $L d+P$ L

$20430 R=S Q R(L 1 * L 1+L 2 * L 2)$

20440 IF R=0 THEN CPdef 1

20450 INPUT "ANGULO CENTRAL RECORRID

20470 INPUT "NUMERO DE TH

20480 FOR $J=1$ TO N1

$20500 \quad x \vee g(N u g+J, 2)=L b+R * \operatorname{SIN}(L d+J * L C$

20510 NEXT J

20520 Nug=Nug+N1

20540 CP7: PRINTER IS 701

20540 CP7: PRINTER IS

20560 PRINT "COORDENADAS DE LOS PUNTOS DEL CONTOPND PLANO"

20570 PRINT USING " $, 5 \mathrm{~A}, 2 \mathrm{X}, 12 \mathrm{~A}, 2 \mathrm{X}, 12 \mathrm{~A}, \boldsymbol{\prime}^{\prime}$; "PUNTO" , "COORDENADA X" , "COORDENADG Y" 20580 PRINT CHRS (27)\&"\&11L"

20600 PRINT USING "50,2X,80.20,2X,80.20" ; J, Xug ( J , 1), Xug ( J , 2

20610 NEXT

20620 PRINT USING "

20630 PRINTER IS

20650 CPB:FOR J=NUG TO 2 STEP -

20660 La $=x \cdot v(J, 1)-X \cup g(J-1,1)$

20670 Lb $=x \vee g(J, 2)-X v g(J-1,2)$

20680 IF SOR $(L a * L a+L b * L b)<1$. E-6 THEN

$20690 \quad$ Nug $=$ Nug -1

$20700 \quad$ FOR $K=J$ TO Nug
$20710 \quad X u g(K, 1)=X v g(K+1,1)$
$20720 \quad X v g(K, 2)=X v g: K+1,2)$

20730 NEXT K

20740 END IF

20760 IF NUG \& 2 THEN RETURN

2070 IF NUg 2 THEN RETURN

(20780 IPUT "COORDENDAS GLOBALES DEL ORIGEN", Xä:*

(NAUT "COORDENADAS DE UN PUNTD DEL EJE X POSITIUT"

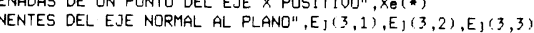

20810 IF R*L*M=0

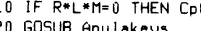

20830 RETURN

\section{publicación del i.e.t.c.c.}

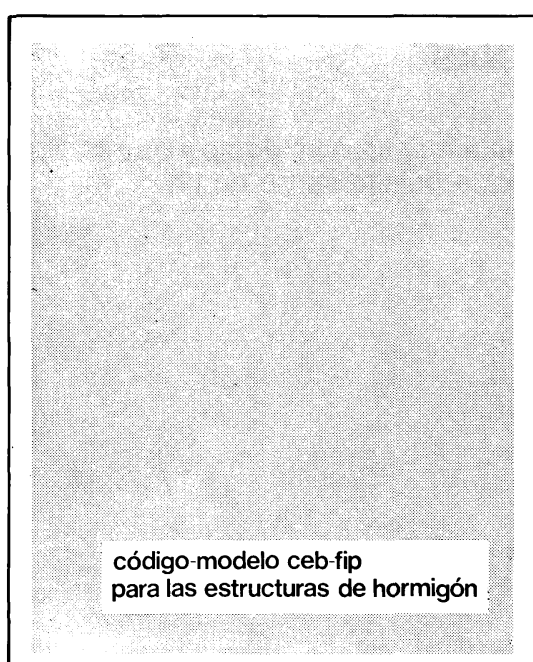

El Instituto Eduardo Torroja, miembro activo tanto del Comité Eurointernacional del Hormigón (CEB), como de la Federación Internacional del Pretensado (FIP), ha tomado a su cargo la traducción y edición de esta importante normativa.

Aunque presentado con el título de "Código Modelo CEB/FIP 1978" este documento incorpora los dos primeros volúmenes de este "Sistema Unificado Internacional de Reglamentación Técnica de Ingenieria Civil». El primer volumen de este "Sistema Unificado" es el denominado "Reglas comunes Unificadas para los diferentes tipos de obras y materiales", donde se exponen los criterios y formatos de seguridad a que han de ajustarse los diferentes de madera), que han de configurar la totalidad del antedicho sistema.

El segundo volumen es propiamente el Código Modelo para las Estructuras de Hormigón. Fruto de la colaboración de dos asociaciones del prestigio del CEB y la FIP, desde mediados de los 60, incorpora los avances cientificos y tecnológicos producidos en los últimos años sin detrimento alguno de la claridad y operatividad que deben presidir un código que pretende ser, ante todo, un auxiliar práctico para los técnicos de la construcción.

El Código sigue en su estructura las reglas más o menos clásicas: una primera parte dedicada a los datos generales para el cálculo (propiedades de los materiales, datos relativos al pretensado, tolerancias); en segundo lugar se presentan las reglas de proyecto estructural (acciones, solicitaciones, estados limites últimos y de utilización, reglas de detalle para el armado); y, por último, ejecución, mantenimiento y control de calidad.

También incluye reglas para estructuras con elementos prefabricados y estructuras de hormigón con áridos ligeros. Los Anejos del Código se refieren a: terminologia, proyecto mediante la experimentación, resistencia al fuego, tecnologia del hormigón, comportamiento en el tiempo del hormigón y fatiga.

Un volumen encuadernado en cartoné, de $21 \times 30 \mathrm{~cm}$, compuesto de 340 páginas, Madrid, mayo 1982.

Precios: España 2.500 ptas. Extranjero 36 \$ USA. 